\title{
Crystal structure of the ubiquitin-like domain of human TBK1
}

\author{
Jian $\mathrm{Li}^{1,3}$, Jun $\mathrm{Li}^{1,3}$, Andrea Miyahira ${ }^{2}$, Jian Sun ${ }^{1,3}$, Yingfang Liu ${ }^{1}$, Genhong Cheng ${ }^{2}$, Huanhuan Liang ${ }^{1 凶}$ \\ ${ }^{1}$ State Key Laboratory of Biomacromolecules, Institute of Biophysics, Chinese Academy of Sciences, Beijing 100101, China \\ 2 Department of Microbiology, Immunology \& Molecular Genetics, University of California, Los Angeles, CA 90095, USA \\ ${ }^{3}$ Graduate School of the Chinese Academy of Sciences, Beijing 100101, China \\ $\square$ Correspondence: hhliang@moon.ibp.ac.cn
}

Received March 29, 2012 Accepted March 31, 2012

\begin{abstract}
TANK-binding kinase 1 (TBK1) is an important enzyme in the regulation of cellular antiviral effects. TBK1 regulates the activity of the interferon regulatory factors IRF3 and IRF7, thereby playing a key role in type I interferon (IFN) signaling pathways. The structure of TBK1 consists of an $\mathrm{N}$-terminal kinase domain, a middle ubiquitin-like domain (ULD), and a C-terminal elongated helical domain. It has been reported that the ULD of TBK1 regulates kinase activity, playing an important role in signaling and mediating interactions with other molecules in the IFN pathway. In this study, we present the crystal structure of the ULD of human TBK1 and identify several conserved residues by multiple sequence alignment. We found that a hydrophobic patch in TBK1, containing residues Leu316, Ile353, and Val382, corresponding to the "lle44 hydrophobic patch" observed in ubiquitin, was conserved in TBK1, IKB kinase epsilon (IKKE/IKKi), IKB kinase alpha (IKKa), and IKB kinase beta (IKKß). In comparison with the structure of the IKK $\beta$ ULD domain of Xenopus laevis, we speculate that the lle44 hydrophobic patch of TBK1 is present in an intramolecular binding surface between ULD and the C-terminal elongated helices. The varying surface charge distributions in the ULD domains of IKK and IKK-related kinases may be relevant to their specificity for specific partners.
\end{abstract}

KEYWORDS TBK1, ubiquitin-like domain, crystal structure, hydrophobic patch

\section{INTRODUCTION}

The TRAF family member-associated NF-kappa-B activator
(TANK)-binding kinase 1 (TBK1, also referred to as NAK or T2K) was initially discovered by yeast 2-hybrid screening by using the TANK protein as a bait and was found to be a nuclear factor (NF)-KB-activating kinase (NAK) homologous to the IKB kinases IKKa and IKK $\beta$, which are well known as the key kinases involved in the regulation of NF-KB activation (Pomerantz and Baltimore, 1999). However, the functions of TBK1 and its close homolog IKB kinase epsilon (IKKE, also known as IKKi) were obscure until it was reported that the TBK1/IKKi complex plays an important role in the induction of type I interferon (IFN) expression (Fitzgerald et al., 2003). There are at least 2 pathways that can activate the TBK $1 / \mathrm{IKKi}$ complex. One is through the regulatory proteins toll-like receptor 3/4 (TLR3/TLR4), TIR domain-containing adapter molecule 1 (TICAM-1 or TRIF), and TNF receptor-associated factor 3 (TRAF3). The second is through retinoid-inducible gene 1 (RIG-1)-like receptors (RLRs), interferon $\beta$ promoter stimulator protein 1 (IPS1; also known as MAVS, VISA, Cardif), mediator of IRF3 activation (MITA; also known as STING) and TRAF3 (Baccala et al., 2007). The TLR3/4 and RLRs belong to the pattern recognition receptor (PRR) family, which recognizes pathogen-associated molecular patterns (PAMPs) and mediates innate immune responses against invading microorganisms (Chen et al., 2009). Upon activation, TBK1/IKKi phosphorylates the IFN regulatory factors IRF3 and IRF7 (IRF3/7), leading to their translocation into the nucleus and activation of type I IFN expression (Tamura et al., 2008). The IFNs and their regulated downstream gene products mediate the inhibition of viral replication, clearance of virus-infected cells, and enhancement of immune responses (Zhong et al., 2008).

Because TBK1 is the key player in this cellular antiviral effect, some viruses have developed defensive mechanisms by antagonizing TBK1 function. For example, $\mathrm{y}_{1} 34.5$ protein of herpes simplex virus type 1 associates with TBK1 and inhibits 
TBK1-mediated phosphorylation of IRF3, thereby replicating more efficiently in host cells (Verpooten et al., 2009). In addition to the induction of IFNs, TBK1 possesses various additional functions such as promoting antibacterial autophagy by phosphorylation of the autophagy receptor optineurin (Wild et al., 2011), attenuating retroviral budding by phosphorylation of the endosomal sorting complex required for transport-I (ESCRT-I) subunit VPS37C (Da et al., 2011), and involvement in malignant transformation (Shen and Hahn, 2011). Thus, the structural determination of this molecule would greatly assist in understanding its functional mechanisms.

The IKKa/IKK $\beta$ and IKK-related kinases TBK1/IKKi are both members of the IKK kinase family. While IKKa and IKK $\beta$ share a $52 \%$ overall homology with one another, TBK1 and IKKi share a $61 \%$ overall homology with one another. However, the homology between classical IKK kinases and IKK-related kinases is limited, with $27 \%$ overall homology and $33 \%$ homology within the catalytic kinase domain (Hiscott, 2007). Hence, substantial structural difference may exist between the two families. Recently, $X u$ et al. reported the crystal structure of Xenopus laevis IKK $\beta(x \mid K K \beta)$ without the C-terminal NEMO-binding domain ( $X u$ et al., 2011). The $\mathrm{xIKK} \beta$ has a $72 \%$ sequence identity with human IKK $\beta$. It has a trimodular architecture comprising a kinase domain, a ubiquitin-like domain (ULD), and an elongated $\alpha$-helical scaffold/dimerization domain (SDD).

Bioinformatic analysis suggests that TBK1 folds into a 3-domain structure similar to that of IKK : an $\mathrm{N}$-terminal Ser/Thr kinase domain, a middle ULD, and C-terminal elongated helices (Fig. 1A). The ULDs are reported to have a variety of functions, including sulfur transfer (Hochstrasser, 2009) and signal transduction (Welchman et al., 2005). Moreover, many proteins in the ubiquitination/proteasomal delivery system and from the deubiquitinase family possess one or more ULDs. One of the well-defined functions of ULDs is to mediate protein-protein interactions in signal transduction and proteasomal delivery (Welchman et al., 2005). It has been reported that the double mutation L352A/I353A in the ULD prevents TBK1 from activating IFN- $\beta$ or RANTES promoters (Ikeda et al., 2007). In all these IKKs and IKK-related kinases, deletion of the ULD results in the loss of the kinase activity (May et al., 2004; Ikeda et al., 2007; Xu et al., 2011), suggesting that ULD is absolutely required for the kinase domain to form an enzymatically active conformation. Hence, ULD is an indispensable part of TBK1; however, the underlying mechanism remains unknown.

In this study, we describe the crystal structure of the ULD of human TBK1. We compared this domain with some structurally similar domains, identified several specific and conserved residues by multiple sequence alignment, and analyzed the surface properties of this domain. Our structure provides detailed information about ULD-mediated potential intra- and inter-molecular interactions between TBK1 and other IKK-related kinases.
A

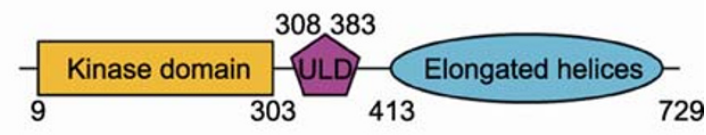

B
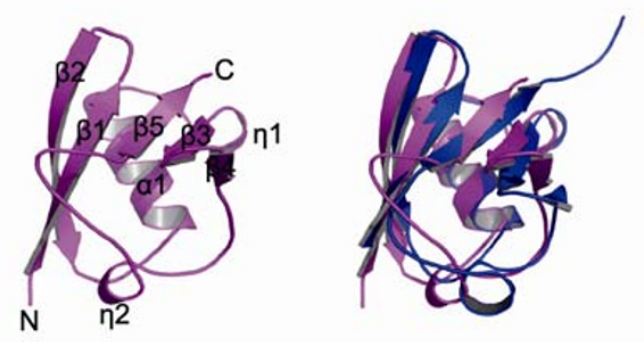

C

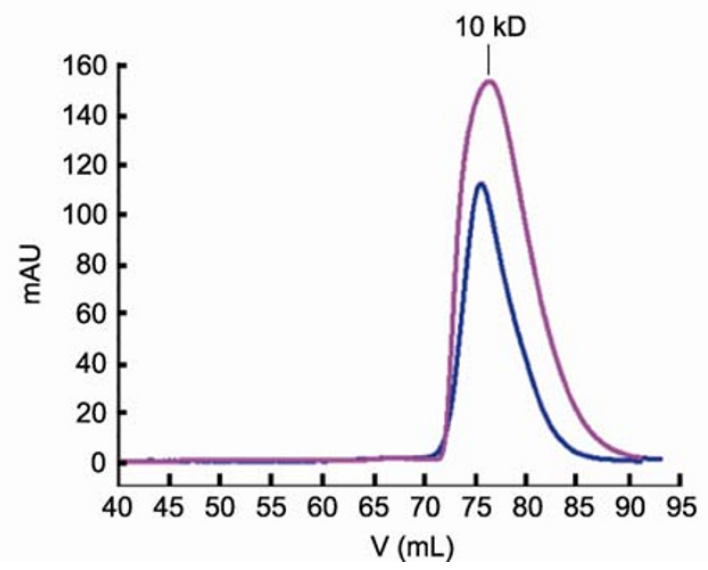

Figure 1. Overall structure of human TBK1 ubiquitin-like domain (ULD). (A) Linear representation of the 3 regions of the TBK1 molecule. (B) A graphical representation of TBK1 ULD (left panel, magenta) and its comparison with human ubiquitin (right panel, blue). The N-terminal, C-terminal, and secondary structural elements of TBK1 ULD are labeled. (C) Gel filtration profile of ULD (magenta) and ubiquitin (blue), showing that they have approximately the same elution volume.

\section{RESULTS}

\section{Overall structure}

We overexpressed human TBK1 ULD covering amino acids 302-383 and finally obtained its crystals (Fig. 1) (see MATERIALS AND METHODS). The crystal adopts a P2(1) space group, with two molecules in an asymmetric unit; its structure was determined at $1.8 \AA$ resolution with the single-wavelength anomalous diffraction (SAD) method by using a selenomethionyl derivative crystal. A summary of crystallographic data and refinement statistics data is shown in Table 1. The structure of ULD contains $5 \beta$ strands and $1 \alpha$ helix. A Dali search (Holm and Rosenström, 2010) from Protein Data 
Table 1 Statistics of crystallographic analysis

\begin{tabular}{|c|c|c|}
\hline Data collection & & \\
\hline Crystal & Native & SeMet \\
\hline Space group & $\mathrm{P} 21$ & $\mathrm{P} 21$ \\
\hline \multicolumn{3}{|l|}{ Cell dimension } \\
\hline$a, b, c(\AA)$ & $\begin{array}{l}34.353,47.785 \\
61.832\end{array}$ & $\begin{array}{l}34.444,47.808, \\
61.934\end{array}$ \\
\hline$\alpha, \beta, \gamma\left({ }^{\circ}\right)$ & $\begin{array}{l}90.000,93.535 \\
90.000\end{array}$ & $\begin{array}{l}90.000,93.346, \\
90.000\end{array}$ \\
\hline Resolution $(\AA)$ & $\begin{array}{l}50-1.77 \\
(1.77-1.80)^{*}\end{array}$ & $\begin{array}{l}50-1.70 \\
(1.70-1.73)\end{array}$ \\
\hline$R_{\mathrm{meg}}$ & $0.081(0.426)$ & \\
\hline $\mid / \sigma l$ & $23.8(4.9)$ & $16.3(2.3)$ \\
\hline Completeness (\%) & $99.8(97.0)$ & $99.9(99.1)$ \\
\hline Redundancy & $7.3(6.0)$ & $7.2(5.2)$ \\
\hline \multicolumn{3}{|l|}{ Refinement } \\
\hline Resolution $(\AA)$ & 1.8 & \\
\hline $\begin{array}{l}\text { Total No. reflection/ } \\
\text { free }\end{array}$ & $19627 / 1004$ & \\
\hline$R_{\text {work }} / R_{\text {free }}$ & $0.170 / 0.204$ & \\
\hline $\begin{array}{l}\text { r.m.s.d. bonds/angles } \\
(\AA)\end{array}$ & $0.006 / 1.015$ & \\
\hline Protein/solvent atoms & $1401 / 214$ & \\
\hline Average B-factors $\left(\AA^{2}\right)$ & 19.23 & \\
\hline \multicolumn{3}{|c|}{ Ramachandran plot statistics } \\
\hline Most favorable & $98.8 \%$ & \\
\hline Additionally allowed & $1.2 \%$ & \\
\hline Disallowed & $0 \%$ & \\
\hline
\end{tabular}

Bank (PDB, http://www.pdb.org) identified hundreds of structures, predominantly the ULDs, similar to TBK1 ULD. The TBK1 ULD structure has a main chain root mean squared deviation of $2.3 \AA$ with human ubiquitin (PDB: 1ubq) (Fig. 1B). Similar to ubiquitin, the ULD of human TBK1 adopts a $\beta$-grasp fold, with $5 \beta$ strands packed against an $\alpha$ helix (Burroughs et al., 2007). The $\beta 1$ (R308-S315) and $\beta 5$ (I379-S383) strands are adjacent and parallel to one another, forming the inner 2 strands. The $\beta 2(\mathrm{M} 319-\mathrm{H} 327)$ strand flanks $\beta 1$, and the $\beta 3$ (Q350-I353) strand flanks $\beta 5$ on either side. Together with $\beta 4$ (R357-L359), these structures form the outer strands. These 5 strands grasp a 12-residue a1 helix (A332-T343), which forms the conserved hydrophobic core of ULD. A long and primarily unstructured region (Y354-P378) connecting $\beta 3$ and $\beta 5$ covers the bottom part of this motif (Fig. 1B).

It has been suggested that TBK1 and IKKi can form both homodimers and heterodimers (Chau et al., 2008). During protein purification of TBK1 ULD with gel filtration, we observed that the elution volume of TBK1 ULD was the same as that of ubiquitin, which exists as a monomer in solution (Bolton et al., 2001) (Fig. 1C). Therefore, we propose that TBK1 ULD also exists as a monomer in solution. Although the
ULDs have been reported to mediate protein-protein interactions in signal transduction, this observation indicates that the TBK1 ULD cannot form homodimers in solution. Therefore, we suggest that the formation of TBK1 homodimer is not mediated by the ULD alone.

\section{The conserved Ile44 hydrophobic patch}

A Dali search (Holm and Rosenström, 2010) from PDB showed that the TBK1 ULD was similar to ULDs that broadly exist in different functional proteins. The ULDs can be classified into 2 categories: small ubiquitin-like modifiers and ULD containing multi-domain proteins. There are at least 10 types of ubiquitin-like modifiers such as Nedd8, SUMO, Fat10, and ISG15 (Hochstrasser, 2009). The integral ULDs can be further divided into several subfamilies: UBX, a module possessed by some proteasomal shuttle factors; PB1 (Phox and Bem1), which heterodimerize by electrostatic force to form a functional signaling complex; Ras-binding domain, which binds and regulates Ras through its $\beta 1$ and $\beta 2$ strands; DCX of doublecortin protein, which binds microtubules; and classic ULD, such as hPLIC_N (human homologs of the yeast ubiquitin-like Dsk2 protein), Rad23N, and NIRF_N (amino-terminal ULD of NP95 and NIRF) (Buchberger et al., 2001; Lytle et al., 2004; Grabbe and Dikic, 2009).

Of these subfamilies, we selected 5 representative structures that were structurally similar to TBK1 ULD and analyzed their sequence and structural characteristics. These structures included human ubiquitin (PDB 1ubq), Nedd8 (PDB $1 \mathrm{r} 4 \mathrm{~m}$ ), the ULD of hHR23A (PDB 1qze), the ULD of NP95 (PDB 2faz), and the ULD of yeast Dsk2 (PDB 2bwf). Four of these form complex structures with their binding partners, which may provide further information about protein interaction sites.

Structure-based sequence alignment of these proteins reveals several conserved residues of TBK1 ULD, including lle311, Val313, Leu316, lle324, lle326, Val339, Ile345, Gln350, Leu352, Ile353, Ile379, Val381, and Val382 (Fig. 2A). Alignment of TBK1 and IKKi in various species showed additional conserved residues, including Phe314, Phe335, and Pro378 (data not shown). Of these conserved residues, Ile311, Val313, lle324, Ile326, Phe335, Val339, lle345, Leu352, Pro378, lle379, and Val381 show very low percentages of solvent-accessible surface areas, suggesting that these residues are primarily structural in nature and contribute to the hydrophobic core of this ubiquitin-like fold (Fig. 2B).

The surface residues Leu316, Ile353, and Val382 are distinct from the hydrophobic core residues and may be involved in protein-protein interactions between ULDs and their binding partners. Interestingly, Leu316, Ile353, and Val382 of TBK1 ULD correspond to the hydrophobic patch formed by Leu8, Ile44, and Val70 in ubiquitin that is involved in interaction with ubiquitin-binding partners (Hurley et al., 2006) (Fig. 2A). In the case of ubiquitin, the majority of ubiquitin-binding 
A

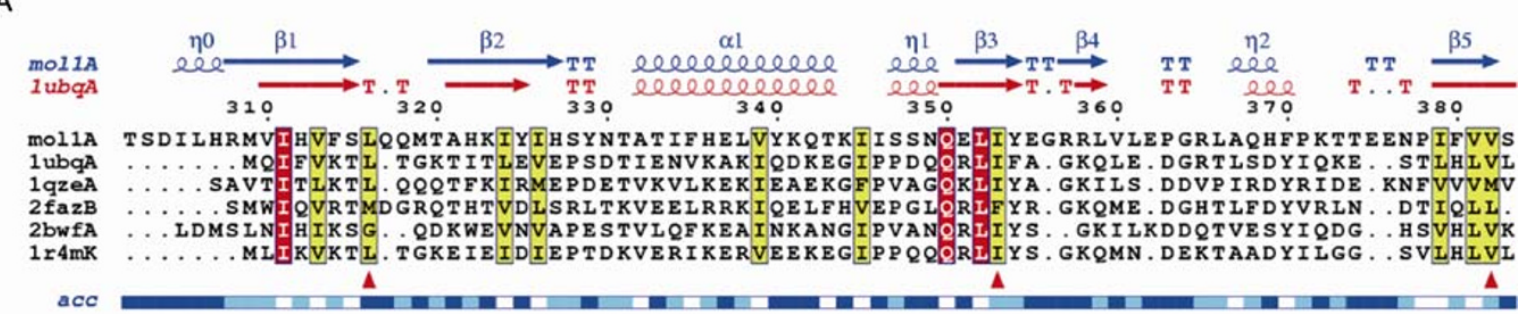

B

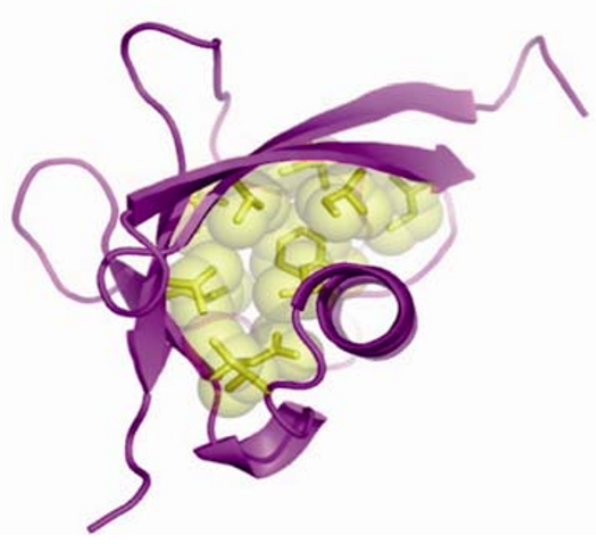

C

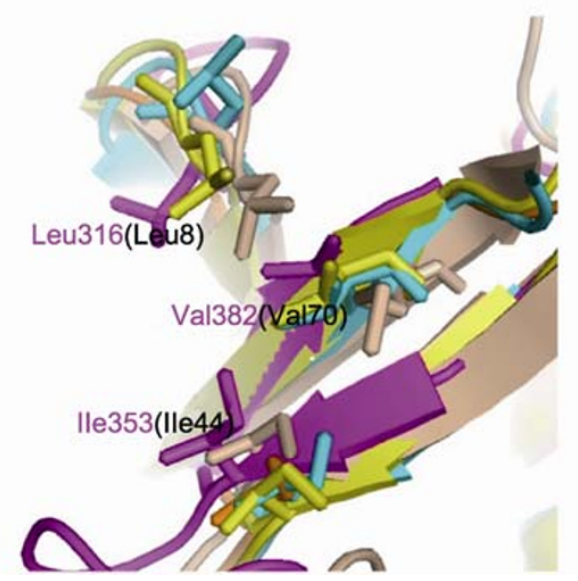

Figure 2. Sequence alignment shows conserved surface residues. (A) Structure-based sequence alignment of mol1A (TBK1 ULD chain a), 1ubqA (ubiquitin), 1qzeA (a UV excision repair protein Rad23 homolog), 2fazB (ubiquitin-like, containing phd and ring finger), 2bwfA (Dsk2), 1r4mK (Nedd8). Secondary structural elements are marked at the top of the sequence alignment. Identical residues are highlighted in red, and conserved residues are highlighted in yellow. Residues forming lle44 hydrophobic patch are marked with red triangles. The bottom blue bar shows relative surface accessibility per residues (white to blue represent increasing surface accessibility). (B) Hydrophobic core of TBK1 ULD, with these residues depicted as semi-transparent spheres. The TBK1 ULD is shown in magenta, and the hydrophobic core residues are shown in yellow. (C) Structure alignment of TBK1 ULD (magenta), ubiquitin (yellow), hHR23A (wheat), Dsk1 ULD (orange), and Nedd8 (cyan) showing the conserved hydrophobic patch corresponding to ubiquitin Leu8, lle44, and Val70.

domains-e.g. UIM, CUE, UBA, GAT, NZF, and UEV domains-bind to ubiquitin through the hydrophobic patch centered on Ile44, known as the Ile44 hydrophobic patch (Hurley et al., 2006). Moreover, deubiquitinases (DUBs) interact with ubiquitin through the lle44 patch (Komander et al., 2009). Of the 5 identified homologous structures, hHR23A, yDsk2, and Nedd8 possess the same lle44 hydrophobic surface patch as that present in ubiquitin and use this region to interact with their corresponding binding partners S5a-UIM, Ufd2, and UBA3, respectively (Mueller and Feigon, 2003; Walden et al., 2003; Hanzelmann et al., 2010). Structural alignment shows that these 3 residues (Leu, Ile, and Val) have similar distributions on the surface of ULDs (Fig. 2C). Based on these results, we suggest that the lle44 hydrophobic patch, including Leu316, Ile353, and Val382, on TBK1 ULD, should be considered potential protein-binding site.

\section{The lle44 hydrophobic patch of TBK1 ULD may mediate the ULD-SDD interaction}

Recently, the crystal structure of $X$. laevis IKK $\beta$ (xIKK $)$ was reported (Xu et al, 2011). The structure comprises 3 domains: a kinase domain, a ULD domain, and an SDD domain. The ULD maintains extensive interactions with SDD, specifically using the lle44 hydrophobic patch to interact with the $\alpha$ helices of SDD (Xu et al, 2011). We aligned the ULD sequences from human TBK1, IKKa, IKK $\beta$, and IKKi with $X$. laevis IKK $\beta$ and found that most residues involved in the ULD-SDD interactions of $x \mathrm{IKK} \beta$, especially the residues corresponding to the lle44 hydrophobic patch, were also conserved in the protein homologs (Fig. 3A). In TBK1, residues F314 and F380 lie adjacent to Leu316, Ile353, and Val382. Together, they form a hydrophobic area comparable to that in $\mathrm{xIKK} \beta$ ULD. It has been reported that the L352A/I353A double mutant of TBK1 ULD fails to interact with the full-length TBK1 and cannot activate the IFN $\beta$ promoter (Ikeda et al., 2007). Considering the significant homology between IKK and IKK-related kinase, we speculate that residues Leu316, Ile353, and Val382 comprising the lle44 hydrophobic patch of TBK1 ULD also play a role in the ULD-SDD interaction in the TKB1 molecule (Fig. 3B). 


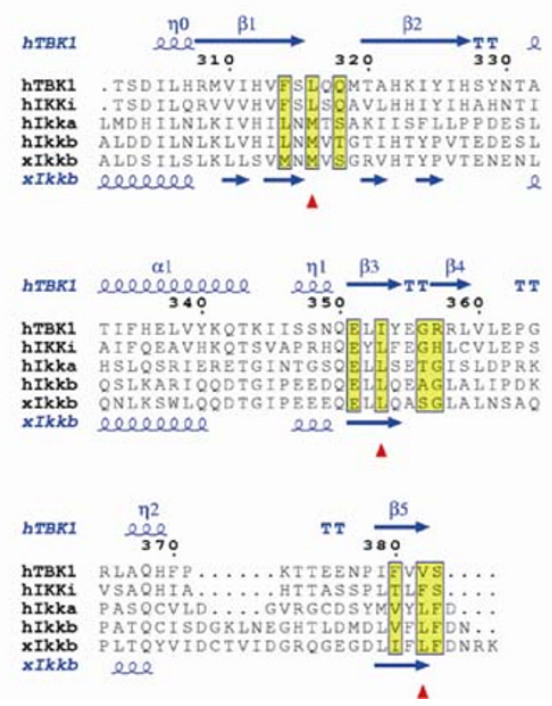

B

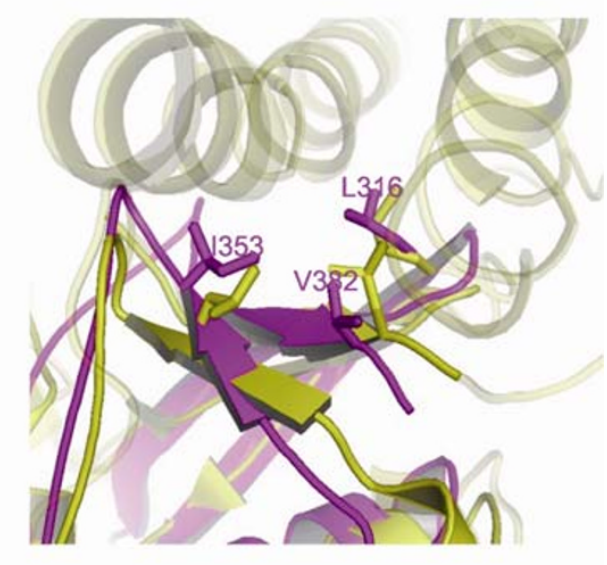

Figure 3. The lle44 hydrophobic patch of TBK1 ULD can provide TBK1 ULD-SDD interface similar to that observed in $\mathbf{x I K K} \beta$. (A) Sequence alignment of IKK and IKK-related kinase ULD showing conserved residues involved in SDD interaction. $\mathbf{x I K K} \beta$ ULD-SDD interaction residues are highlighted in yellow. Residues forming the lle44 hydrophobic patch are marked with red triangles. (B) Distribution of the lle44 patch residues (shown as sticks) on TBK1 ULD surface (magenta). xIKK $\beta$ ULD (yellow) was aligned to TBK1 ULD structure. lle44 patch residues of xIKK $\beta$ ULD involved in ULD-SDD interaction are shown as sticks.

\section{Surface charge comparison of ULD between IKK and IKK-related kinase}

Because the ubiquitin-like domain is usually a protein-protein interaction motif (May et al., 2004; Ikeda et al., 2007; Xu et al., 2011) and the IKK and IKK-related kinase possess different binding partners in the innate immune pathways, we compared their ULDs to examine the structural mechanism behind their specificities. Because human IKKi ULD shares a $50 \%$ sequence identity with TBK1, we performed homology modeling of IKKi ULD by using our structure as the template in the Swiss-Model web server (Guex and Peitsch, 1997; Schwede et al., 2003; Arnold et al., 2006). We also modeled the ULD structures of human IKK 3 and IKKa by using the $x \mid K K \beta$ ULD structure, because $x$ IKK $\beta$ ULD has $57 \%$ and $32 \%$ sequence identity with the ULD of human IKK $\beta$ and IKKa, respectively. Subsequently, we analyzed and compared the different features of electrostatic surfaces between these ULDs (Fig. 4).

We observed a prominent positively charged area on one of the surfaces of TBK1/IKKi (Fig. 4I\&J, highlighted in black circles), whereas the homologous region of IKKa/IKK $\beta$ was quite different (Fig. 4G\&H). A conserved Arg (Arg308 in TBK1 and Arg308 in IKKi) from different species of TBK1/IKKi on the $\beta 1$ strand contributes to this positively charged area. In contrast, IKKa/IKK $\beta$ shows prominent negative charges on the loop connecting the $\beta 2$ strand and the $\alpha 1$ helix where several negatively charged Glu and Asp residues are situated (Asp 331 and Glu 332 in IKKa and Glu 329, Asp330, and
Glu331 in IKKß) (Fig. 4G\&H, highlighted in black circles).

We also observed a negatively charged surface patch in all these ULDs when viewing the structure from the C-terminus of $x \mid K K \beta$ SDD (Fig. 4K-O, highlighted in black circles). This patch corresponds to the amino acid sequence surrounding $\beta 3$ where Glu (Glu351 in TBK1) contributes to a portion of this negative charge. Because the amino acid sequence " ${ }^{350} \mathrm{QELI}^{353 "}$ in the $\beta 3$ strand of TBK1 ULD is conserved in TBK1, IKKi, IKKB, and IKKa (Fig. 3A), we suggest that this negative charge may have additional, unknown functions.

\section{DISCUSSION}

In this study, we present the crystal structure of the TBK1 ULD. Based on sequence and structural analysis, we showed that TBK1 ULD has a ubiquitin-like structure and an Ile44 hydrophobic patch, which is conserved among ULDs and IKK and IKK-related proteins. Analysis of the published complex structures in PDB showed that the exposed face of the $\beta$-sheets in the lle44 patch mediates a greater number of interactions across the $\beta$-grasp fold (Burroughs et al., 2007). The results of our sequence and structural alignment with $\mathrm{xIKK} \beta$ suggest that this hydrophobic patch is involved in ULD-SDD interactions in TBK1 and other IKK and IKK-related proteins. Because many protein domains UIM, UBA, CUE, GAT (Hurley et al., 2006) and NEMO (IKKy) use their a helical regions to bind to ubiquitin (Rahighi et al., 2009), it is not surprising that the ULDs tend to bind the 
A

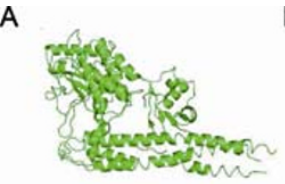

F
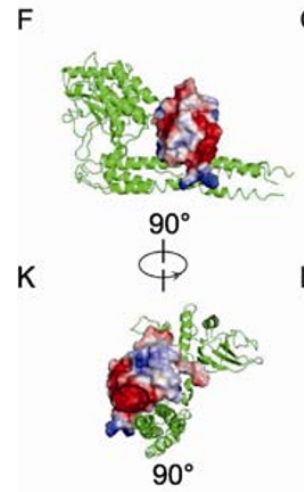

P

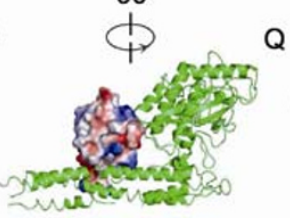

B

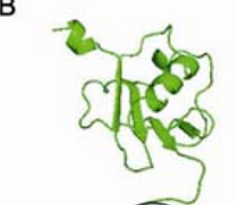

G

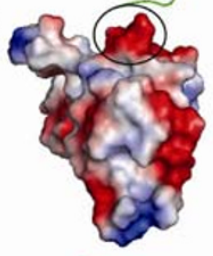

L
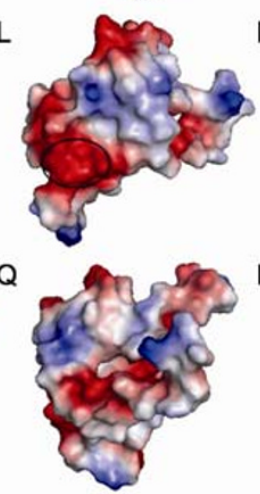

C

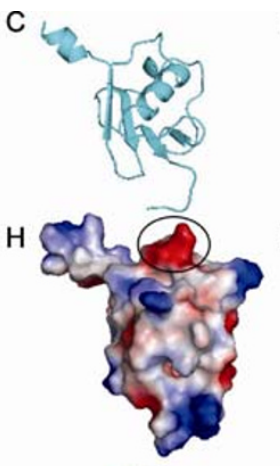

D
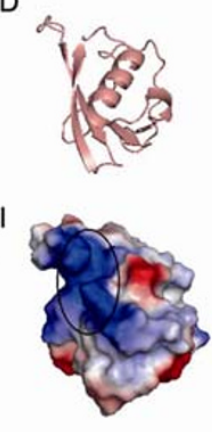

M
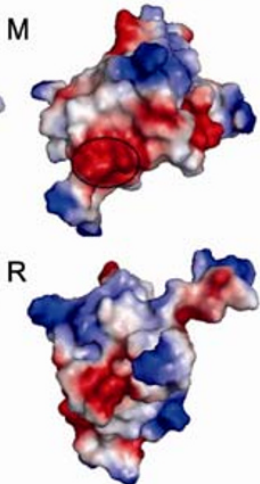

N

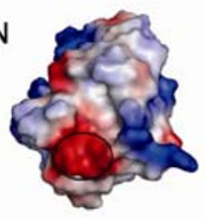

$\mathrm{S}$

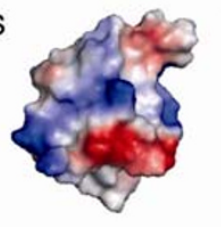

E

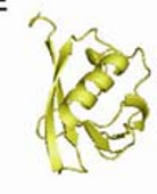

J

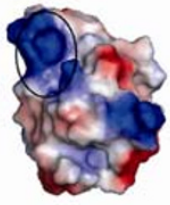

O

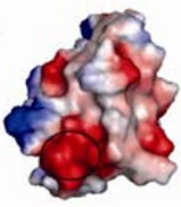

T

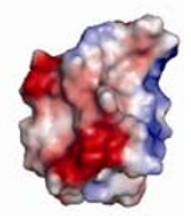

Figure 4. ULD surface charge distribution of IKK and IKK-related kinase (red, negative charge; blue, positive charge; white, neutral). (A, F, K, P): (A) Structure of Xenopus laevis IKK 3 . (F) Same view as in (A) with ULD surface charge displayed. (K) After rotating $90^{\circ}$ around the longitudinal axis, i.e. viewing from SDD tail. (P) After rotating another $90^{\circ}$ around the longitudinal axis, i.e. the opposite surface compared to $(F)$. $(B, G, L, Q)$ : Surface charge distribution of human IKK $\beta$ ULD homology model, corresponding view to $(A),(F),(K)$, and $(P)$, respectively. $(C, H, M, R)$ : Surface charge distribution of human IKK $\alpha$ ULD homology model, corresponding view to $(A),(F),(K)$, and $(P)$, respectively. $(D, I, N, S)$ : Surface charge distribution of human TBK1 ULD, corresponding view to $(A),(F),(K)$, and $(P)$ respectively. $(E, J, O, T)$ : Surface charge distribution of human IKKi ULD homology model, corresponding view to $(A),(F),(K),(P)$ respectively. The positively charged surface patch in $(I)$ and $(J)$ and the negatively charged surface patch in $(\mathrm{G}),(\mathrm{H}),(\mathrm{K}),(\mathrm{L}),(\mathrm{M}),(\mathrm{N})$, and $(\mathrm{O})$ are highlighted in black circles.

a-helical SDD.

To date, research on the use of ULD as an interaction module is supported by the following findings: (1) IKK $\beta$ ULD binds weakly to its specific substrate IKB (Xu et al., 2011), (2) ULD of human IKK $\beta$ alone can associate with Homer 3 coiled-coil/leucine zipper domain (Yatherajam et al., 2010), and (3) TBK1 ULD interacts with its substrate (IRF3) through the IAD domain of IRF3 (Ikeda et al., 2007). Hence, there must exist additional binding surfaces apart from the conserved lle44 hydrophobic patch described above. We analyzed the electrostatic surfaces of the ULDs in IKK and IKK-related proteins by performing a structural alignment (Fig. 4) and observed several conserved and differential features that may be relevant to IKK specificities for their interaction partners. Moreover, after an extensive investigation of the known structures of ULD complexes with their binding partners in PDB, 3 distinct regions on ULD emerge as hotspots for protein interaction (Fig. 5) in addition to the Ile44 patch on the exposed face of $\beta$ sheets. These 3 areas correspond to the 3 modes of interaction that are compatible with TBK1 ULD. In the first type of interaction, ULD uses its $\beta 1$ strand to form a continuous intermolecular $\beta$ sheet with its binding partner (Fig. 5A), represented by RBD-Ras and SUMO-SIM complexes (Nassar et al., 1995; Namanja et al., 2012). In the second type of interaction, the binding site is almost exactly opposite the lle44 hydrophobic patch, comprising the $\alpha 1$ and $\beta 2-\alpha 1$ loop (Fig. $5 \mathrm{~B}$ ). This is observed in the yHub1-HIND structure, in which a negatively charged Asp residue located in the $\beta 2-\alpha 1$ loop forms a salt bridge with HIND (Mishra et al., 2011). Interestingly, IKKa/IKK $\beta$ also display prominent negative charges in the same region, while TBK1/IKKi do not. In the third type of interaction, ubiquitin interacts with an A20 zinc-finger domain (A20 ZnF) through the region between $\beta 4$ and $\beta 5$ (Lee et al., 2006) (Fig. 5C). This region is primarily an unstructured loop, with a length that is different between IKK and IKK-related family members. In the $x I K K \beta$ structure, several residues are missing from the electron-density map, suggesting the unordered nature of this region, which may be involved in interaction with its binding partners. The proposed binding sites are supported by the results from an online web server for the prediction of protein interaction sites PredUs and meta-PPISP (Qin and Zhou, 


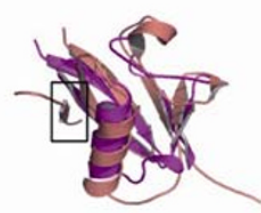

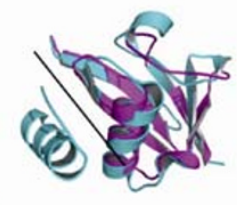

C

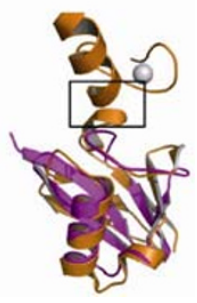

Figure 5. Proposed intermolecular modes of interaction from TBK1 ULD. These 3 modes are exemplified by $(A)$ SUMO-SIM (PDB 2LAS), (B) yHub1-HIND1 (PDB 3PLU), and (C) ubiquitin-A20 ZnF (PDB 2L00) structures, respectively. TBK1 ULD is colored magenta and aligned to the 3 structures, respectively. The interfaces are labeled with a black box or a line.

2007; Zhang et al., 2011). Taken together, we proposed 3 potential intermolecular modes of interaction for TBK1 ULD. Although this hypothesis requires further work, our structure and analysis may pave the way for further efforts to identify and characterize the ULD-partner interaction.

In conclusion, we provide a high-resolution crystal structure of human TBK1 ULD, a pivotal kinase in IFN production, and analyze its special features for its potential interaction. These results would be important for understanding the structural and functional differences of IKK and IKK-related kinase.

\section{MATERIALS AND METHODS}

\section{Protein expression and purification}

Recombinant human TBK1 ULD (amino acids 302-383) was expressed in E. coli as a GST fusion protein using a pGEX-6p-1 vector (GE Healthcare). After induction with $0.2 \mathrm{mmol} / \mathrm{L} \mathrm{IPTG}$ at $16^{\circ} \mathrm{C}$ for 20 $\mathrm{h}$, the cells were harvested and lysed in PBS buffer ( $\mathrm{pH} \mathrm{7.4)} \mathrm{by}$ sonication. The debris and the insoluble fraction were removed by centrifugation at $20,000 \mathrm{~g}$ for $30 \mathrm{~min}$ and the supernatant was loaded onto a glutathione affinity column (GE Healthcare). After extensive washing with PBS buffer, the GST fusion protein was cleaved in situ by PreScission Protease digestion in a buffer containing $50 \mathrm{mmol} / \mathrm{L}$ Tris- $\mathrm{HCl}, 150 \mathrm{mmol} / \mathrm{L} \mathrm{NaCl}, 1 \mathrm{~mol} / \mathrm{L}$ urea, and $5 \%$ glycerol $(\mathrm{pH} 8.0)$. The eluted protein was further purified by $Q$ ion exchange chromatography followed by Superdex-75 gel filtration chromatography (GE Healthcare). Selenomethionine-derivatized protein was purified in a similar manner. The purified protein contains the additional vector-derived amino acid sequence GPLGS at the $\mathrm{N}$ terminus and LERPHRD at the $\mathrm{C}$ terminus.

The budding yeast ubiquitin was expressed in $E$. coli as a his-tagged protein using a $\mathrm{pET}-28 \mathrm{a}$ vector. Yeast ubiquitin was purified by nickel affinity column, and applied to Superdex-75 gel filtration chromatography.

\section{Crystallization}

Purified samples were concentrated to approximately $10 \mathrm{mg} / \mathrm{mL}$ in 50 $\mathrm{mmol} / \mathrm{L}$ Tris- $\mathrm{HCl}, 150 \mathrm{mmol} / \mathrm{L} \mathrm{NaCl}, 1 \mathrm{~mol} / \mathrm{L}$ urea, $5 \%$ glycerol $(\mathrm{pH}$ 8.0). Crystals were grown by the hanging-drop vapor diffusion method at $16^{\circ} \mathrm{C}$ by mixing $1 \mu \mathrm{L}$ protein with $1 \mu \mathrm{L}$ reservoir solution. The best diffracting crystals were obtained in a reservoir solution containing $0.1 \mathrm{~mol} / \mathrm{L}$ BIS-TRIS, $0.2 \mathrm{~mol} / \mathrm{L} \mathrm{NaCl}$, and 21\% PEG3350 (pH 5.3). For selenomethionine-derivatized protein, the best quality crystals were grown in a condition containing $0.1 \mathrm{~mol} / \mathrm{L}$ BIS-TRIS, 0.2 $\mathrm{mol} / \mathrm{L}$ lithium sulfate monohydrate, and 25\% PEG3350 (pH 5.5).

\section{Data collection and structure determination}

Native and SeMet MAD data sets were collected on beamline BL5A (KEK-PF, Japan; $\lambda=0.978 \AA$ ) and BL17U (SSRF, China; $\lambda=0.979$ $\AA$ ), respectively. Data were processed using the HKL2000 program suite (Minor, 1997). The structure of TBK1 ULD was solved by the SAD method. Heavy atom positions were located by SHELXD (Sheldrick, 2008). An initial model was automatically built by Phenix Autosol (Adams et al., 2010), manually modified with Coot (Emsley and Cowtan, 2004), and refined with Phenix Refine. The final model contains no missing fragment and has an $R_{\text {work }}$ of $17.0 \%$ and an $R_{\text {free }}$ of $20.4 \%$. Data scaling, refinement, and validation statistics are shown in Table 1. The atomic coordinates and diffraction data have been deposited in the Protein Data Bank (4EFO). Structural figures were prepared using PyMOL (http://www.pymol.org).

\section{ACKNOWLEDGEMENTS}

We thank colleagues at KEK-PF (Japan) and SSRF (China) for their assistance in the use of the synchrotron resource. This work was supported by grants from the State Key Development Program for Basic Research of the Ministry of Science and Technology of China (973 Program) (Grant Nos. 2011CB910304 and 2012CB910204) to YL and the National Natural Science Foundation of China (Grant Nos. 30925011, 31030024 and 31021062).

\section{ABBREVIATIONS}

IFN, interferon; IPS1, interferon $\beta$ promoter stimulator protein 1; IRF, IFN regulatory factor; NAK, NF-KB-activating kinase; NF, nuclear factor; PAMPs, pathogen-associated molecular patterns; PRR, pattern recognition receptor; RIG-1, retinoid-inducible gene 1; RLRs, RIG1-like receptors; SAD, single-wavelength anomalous diffraction; SDD, scaffold/dimerization domain; TBK1, TANK-binding kinase 1; TLR, toll-like receptor; TRAF3, TNF receptor-associated factor 3; ULD, ubiquitin-like domain

\section{REFERENCES}

Adams, P.D., Afonine, P.V., Bunkoczi, G., Chen, V.B., Davis, I.W., Echols, N., Headd, J.J., Hung, L.W., Kapral, G.J., Grosse-Kunstleve, R.W., et al. (2010). PHENIX: a comprehensive Python-based system for macromolecular structure solution. Acta Crystallogr D Biol Crystallogr 66, 213-221.

Arnold, K., Bordoli, L., Kopp, J., and Schwede, T. (2006). The SWISS-MODEL workspace: a web-based environment for protein structure homology modelling. Bioinformatics 22, 195-201. 
Baccala, R., Hoebe, K., Kono, D.H., Beutler, B., and Theofilopoulos, A.N. (2007). TLR-dependent and TLR-independent pathways of type I interferon induction in systemic autoimmunity. Nat Med 13, 543-551.

Bolton, D., Evans, P.A., Stott, K., and Broadhurst, R.W. (2001). Structure and properties of a dimeric N-terminal fragment of human ubiquitin. J Mol Biol 314, 773-787.

Buchberger, A., Howard, M.J., Proctor, M., and Bycroft, M. (2001). The UBX domain: a widespread ubiquitin-like module. J Mol Biol 307, 17-24.

Burroughs, A.M., Balaji, S., lyer, L.M., and Aravind, L. (2007). Small but versatile: the extraordinary functional and structural diversity of the beta-grasp fold. Biol Direct 2, 18.

Chau, T.L., Gioia, R., Gatot, J.S., Patrascu, F., Carpentier, I., Chapelle, J.P., O’Neill, L., Beyaert, R., Piette, J., and Chariot, A. (2008). Are the IKKs and IKK-related kinases TBK1 and IKK-epsilon similarly activated? Trends Biochem Sci 33, 171-180.

Chen, G., Shaw, M.H., Kim, Y.G., and Nuñez, G. (2009). NOD-like receptors: role in innate immunity and inflammatory disease. Annu Rev Pathol 4, 365-398.

Da, Q., Yang, X., Xu, Y., Gao, G., Cheng, G., and Tang, H. (2011). TANK-binding kinase 1 attenuates PTAP-dependent retroviral budding through targeting endosomal sorting complex required for transport-I. J Immunol 186, 3023-3030.

Emsley, P., and Cowtan, K. (2004). Coot: model-building tools for molecular graphics. Acta Crystallogr D Biol Crystallogr 60, 2126-2132.

Fitzgerald, K.A., McWhirter, S.M., Faia, K.L., Rowe, D.C., Latz, E., Golenbock, D.T., Coyle, A.J., Liao, S.M., and Maniatis, T. (2003). IKKepsilon and TBK1 are essential components of the IRF3 signaling pathway. Nat Immunol 4, 491-496.

Grabbe, C., and Dikic, I. (2009). Functional roles of ubiquitin-like domain (ULD) and ubiquitin-binding domain (UBD) containing proteins. Chem Rev 109, 1481-1494.

Guex, N., and Peitsch, M.C. (1997). SWISS-MODEL and the Swiss-PdbViewer: an environment for comparative protein modeling. Electrophoresis 18, 2714-2723.

Hänzelmann, P., Stingele, J., Hofmann, K., Schindelin, H., and Raasi, S. (2010). The yeast E4 ubiquitin ligase Ufd2 interacts with the ubiquitin-like domains of Rad23 and Dsk2 via a novel and distinct ubiquitin-like binding domain. J Biol Chem 285, 20390-20398.

Hiscott, J. (2007). Convergence of the NF-kappaB and IRF pathways in the regulation of the innate antiviral response. Cytokine Growth Factor Rev 18, 483-490.

Hochstrasser, M. (2009). Origin and function of ubiquitin-like proteins. Nature 458, 422-429.

Holm, L., and Rosenström, P. (2010). Dali server: conservation mapping in 3D. Nucleic Acids Res 38, W545-549.

Hurley, J.H., Lee, S., and Prag, G. (2006). Ubiquitin-binding domains. Biochem J 399, 361-372.

Ikeda, F., Hecker, C.M., Rozenknop, A., Nordmeier, R.D., Rogov, V., Hofmann, K., Akira, S., Dötsch, V., and Dikic, I. (2007). Involvement of the ubiquitin-like domain of TBK1/IKK-i kinases in regulation of IFN-inducible genes. EMBO J 26, 3451-3462.
Komander, D., Clague, M.J., and Urbé, S. (2009). Breaking the chains: structure and function of the deubiquitinases. Nat Rev Mol Cell Biol 10, 550-563.

Lee, S., Tsai, Y.C., Mattera, R., Smith, W.J., Kostelansky, M.S., Weissman, A.M., Bonifacino, J.S., and Hurley, J.H. (2006). Structural basis for ubiquitin recognition and autoubiquitination by Rabex-5. Nat Struct Mol Biol 13, 264-271.

Lytle, B.L., Peterson, F.C., Quu, S.H., Luo, M., Zhao, Q., Markley, J.L., and Volkman, B.F. (2004). Solution structure of a ubiquitin-like domain from tubulin-binding cofactor B. J Biol Chem 279, 46787-46793.

May, M.J., Larsen, S.E., Shim, J.H., Madge, L.A., and Ghosh, S. (2004). A novel ubiquitin-like domain in IkappaB kinase beta is required for functional activity of the kinase. J Biol Chem 279, 45528-45539.

Minor, Z.O.W. (1997). Processing of X-ray Diffraction Data Collected in Oscillation Mode. Methods Enzymol 276, 20.

Mishra, S.K., Ammon, T., Popowicz, G.M., Krajewski, M., Nagel, R.J., Ares, M. Jr, Holak, T.A., and Jentsch, S. (2011). Role of the ubiquitin-like protein Hub1 in splice-site usage and alternative splicing. Nature 474, 173-178.

Mueller, T.D., and Feigon, J. (2003). Structural determinants for the binding of ubiquitin-like domains to the proteasome. EMBO $\mathrm{J} 22$, 4634-4645.

Namanja, A.T., Li, Y.J., Su, Y., Wong, S., Lu, J., Colson, L.T., Wu, C., Li, S.S., and Chen, Y. (2012). Insights into high affinity small ubiquitin-like modifier (SUMO) recognition by SUMO-interacting motifs (SIMs) revealed by a combination of NMR and peptide array analysis. J Biol Chem 287, 3231-3240.

Nassar, N., Horn, G., Herrmann, C., Scherer, A., McCormick, F., and Wittinghofer, A. (1995). The 2.2 A crystal structure of the Ras-binding domain of the serine/threonine kinase c-Raf1 in complex with Rap1A and a GTP analogue. Nature 375, 554-560.

Pomerantz, J.L., and Baltimore, D. (1999). NF-kappaB activation by a signaling complex containing TRAF2, TANK and TBK1, a novel IKK-related kinase. EMBO J 18, 6694-6704.

Qin, S., and Zhou, H.X. (2007). meta-PPISP: a meta web server for protein-protein interaction site prediction. Bioinformatics 23, 3386-3387.

Rahighi, S., Ikeda, F., Kawasaki, M., Akutsu, M., Suzuki, N., Kato, R., Kensche, T., Uejima, T., Bloor, S., Komander, D., et al. (2009). Specific recognition of linear ubiquitin chains by NEMO is important for NF-kappaB activation. Cell 136, 1098-1109.

Schwede, T., Kopp, J., Guex, N., and Peitsch, M.C. (2003). SWISS-MODEL: An automated protein homology-modeling server. Nucleic Acids Res 31, 3381-3385.

Sheldrick, G.M. (2008). A short history of SHELX. Acta Crystallogr A 64, 112-122.

Shen, R.R., and Hahn, W.C. (2011). Emerging roles for the non-canonical IKKs in cancer. Oncogene 30, 631-641.

Tamura, T., Yanai, H., Savitsky, D., and Taniguchi, T. (2008). The IRF family transcription factors in immunity and oncogenesis. Annu Rev Immunol 26, 535-584.

Verpooten, D., Ma, Y., Hou, S., Yan, Z., and He, B. (2009). Control 
of TANK-binding kinase 1-mediated signaling by the gamma(1)34.5 protein of herpes simplex virus 1. J Biol Chem 284, 1097-1105.

Walden, H., Podgorski, M.S., Huang, D.T., Miller, D.W., Howard, R.J., Minor, D.L. Jr, Holton, J.M., and Schulman, B.A. (2003). The structure of the APPBP1-UBA3-NEDD8-ATP complex reveals the basis for selective ubiquitin-like protein activation by an E1. Mol Cell 12, 1427-1437.

Welchman, R.L., Gordon, C., and Mayer, R.J. (2005). Ubiquitin and ubiquitin-like proteins as multifunctional signals. Nat Rev Mol Cell Biol 6, 599-609.

Wild, P., Farhan, H., McEwan, D.G., Wagner, S., Rogov, V.V., Brady, N.R., Richter, B., Korac, J., Waidmann, O., Choudhary, C., et al. (2011). Phosphorylation of the autophagy receptor optineurin restricts Salmonella growth. Science 333, 228-233.
Xu, G., Lo, Y.C., Li, Q., Napolitano, G., Wu, X., Jiang, X., Dreano, M., Karin, M., and $\mathrm{Wu}, \mathrm{H}$. (2011). Crystal structure of inhibitor of $\mathrm{kB}$ kinase $\beta$. Nature 472, 325-330.

Yatherajam, G., Banerjee, P.P., McCorkell, K.A., Solt, L.A., Hanson, E.P., Madge, L.A., Kang, S., Worley, P.F., Orange, J.S., and May, M.J. (2010). Cutting edge: association with I kappa B kinase beta regulates the subcellular localization of Homer3. J Immunol 185, 2665-2669.

Zhang, Q.C., Deng, L., Fisher, M., Guan, J., Honig, B., and Petrey, D. (2011). PredUs: a web server for predicting protein interfaces using structural neighbors. Nucleic Acids Res 39, W283-287.

Zhong, B., Yang, Y., Li, S., Wang, Y.Y., Li, Y., Diao, F., Lei, C., He, X. Zhang, L., Tien, P., et al. (2008). The adaptor protein MITA links virus-sensing receptors to IRF3 transcription factor activation. Immunity 29, 538-550. 